\title{
First results from a multisite campaign on AV Cet
}

\author{
T. H. Dall ${ }^{1}$, G. Handler ${ }^{2,3}$, M. B. Moalusi ${ }^{4}$ \\ ${ }^{1}$ European Southern Observatory, Alonso de Cordova 3107, Casilla 19001, Santiago, \\ Chile \\ 2 Institut für Astronomie, Türkenschanzstrasse 17, 1180 Vienna, Austria \\ 3 South African Astronomical Observatory, P.O. Box 9, Observatory 7935, South \\ Africa \\ ${ }^{4}$ Department of Physics, University of the North-West, Private Bag X2046, \\ Mmabatho 2735, South Africa
}

\begin{abstract}
We present the first results from an international observing campaign on the $\delta$ Scuti star AV Cet. From more than 80 hours of Strömgren photometry, not yet fully analysed, we find 8 frequencies between $14 \mathrm{~d}^{-1}$ and $31 \mathrm{~d}^{-1}$, with amplitudes between $4.4 \mathrm{mmag}$ and $1.6 \mathrm{mmag}$. Among the detected frequencies are the three that were found in previous studies. Results from simultaneous spectroscopy will be used in a later paper to attempt mode identification of the strongest modes of AV Cet.
\end{abstract}

\section{Introduction}

Little work has been done on the star AV Cet (HD 8511, V=6.21, F0V) since it was recognized as a $\delta$ Scuti pulsator by Jørgensen et al. (1971). Based on four nights of photoelectric observations Gonzalez-Bedolla (1990) and GonzalezBedolla et al. (1990) found one dominant frequency of $14.59 \mathrm{~d}^{-1}$ and a suspected second frequency of $19.19 \mathrm{~d}^{-1}$. Later Dall \& Frandsen (2002) verified the frequencies and found evidence for an additional frequency around $33 \mathrm{~d}^{-1}$ in the line indices of the Balmer lines, based on only four hours of spectroscopy.

In order to verify this star as a multimode pulsator and to clarify its pulsational content an international photometric campaign was launched in October 2001, involving sites in Chile, South Africa and Australia. Regrettably we were unable to obtain data from Australia due to dome repair works that took much longer than expected. The observing log is presented in Table 1. 


\begin{tabular}{llrcl}
\hline Observatory & Telescope & $\begin{array}{r}\text { Strömgren } \\
\text { filters }\end{array}$ & $\begin{array}{c}\text { hours } \\
\text { observed }\end{array}$ & HJD - 52200 \\
\hline ESO, La Silla & $0.5 \mathrm{~m}$ SAT & uvby & 41.5 & $8,11,12,13,14,15$ \\
SAAO & $0.5 \mathrm{~m}$ & vby & 42.2 & $8,9,12,13,14,15,16,18$ \\
\hline
\end{tabular}

Table 1: The participating sites and the amount of time used.

Alongside the photometric campaign, spectroscopy was done with the Danish $1.54 \mathrm{~m}$ at La Silla, Chile. We will attempt mode identification by comparing the same modes in photometry and Balmer-line-indices, as has been done for FG Vir (Viskum et al. 1998) and BN Cnc (Dall et al. 2002). The spectroscopic data and part of the photometric data are still undergoing analysis, so here we will only present preliminary results of the photometric campaign.

\section{Data analysis and results}

The raw data from La Silla was fed through the standard reduction pipeline developed by E. H. Olsen et al. at the Copenhagen University Observatory, while the data from South Africa was locally reduced in the standard way. So far, only the Strömgren $y$ data has undergone frequency analysis.

The data set was assigned statistical weights based on the local scatter in the time series with each sub-series, defined by observatory and date, treated individually. The frequency analysis was done using Period98 (Sperl 1998), which yielded the amplitude spectrum of the combined $y$ data set shown in Fig. 1. The frequency content of AV Cet is given in Table 2.

\begin{tabular}{lrrr}
\hline mode & $\begin{array}{r}\text { frequency } \\
{\left[\mathrm{d}^{-1}\right]}\end{array}$ & $\begin{array}{r}\text { amplitude } \\
{[\mathrm{mmag}]}\end{array}$ & $\mathrm{S} / \mathrm{N}$ \\
\hline$f_{1}$ & 15.90 & 4.4 & 19 \\
$f_{2}$ & 14.59 & 2.6 & 11 \\
$f_{3}$ & 28.16 & 2.1 & 8.9 \\
$f_{4}$ & 25.95 & 2.0 & 8.5 \\
$f_{5}$ & 30.83 & 1.7 & 7.2 \\
$f_{6}$ & 29.39 & 1.6 & 6.8 \\
$f_{7}$ & 16.36 & 1.6 & 6.8 \\
$f_{8}$ & 21.20 & 1.6 & 6.8 \\
\hline
\end{tabular}

Table 2: The frequencies found in the combined Strömgren $y$ data set of AV Cet. The frequency spectrum after prewhitening with $f_{1}-f_{8}$ shows excess power, but we prefer not to present any more frequencies before the full data set has been analysed. 

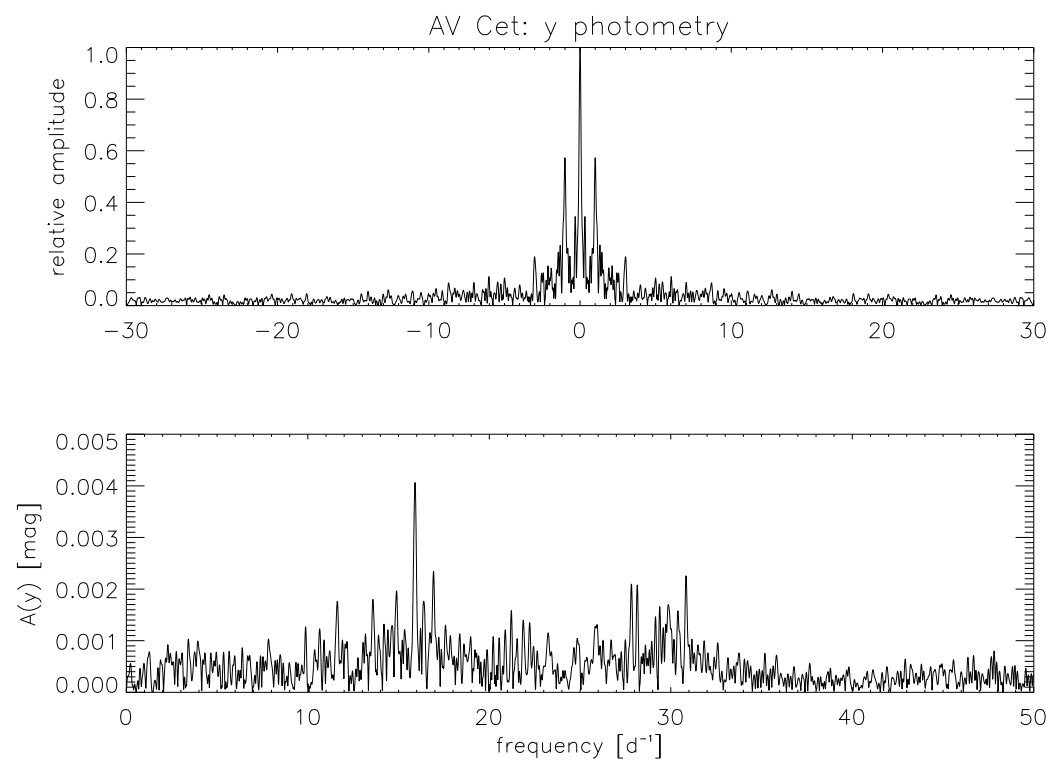

Figure 1: The amplitude spectrum of the combined $y$ data set is shown in the bottom panel, with the window shown in the top panel.

\section{Discussion}

Our preliminary analysis shows already that AV Cet has many pulsational modes excited. In this respect it resembles other extensively studied $\delta$ Scuti stars of low amplitude. We find that the mode $f_{2}$ reported to be the dominant by Gonzalez-Bedolla is exceeded by another mode close by, while the mode reported at $19.19 \mathrm{~d}^{-1}$ might be explained by our $f_{8}$ mode, whose frequency is different by $2.01 \mathrm{~d}^{-1}$, or by a combination of closely spaced frequencies, which are present around $20 \mathrm{~d}^{-1}$.

The variation around $33 d^{-1}$ reported by Dall \& Frandsen from only four hours of observations is found to consist of several close frequencies. The low amplitudes of these signals makes it likely that they are not detectable in the old data of Gonzalez-Bedolla. On the other hand, this region shows larger excess power than the region around $20 \mathrm{~d}^{-1}$, so the possibility of amplitude variations or even excitation of new modes cannot be ruled out.

In a later paper we will present a detailed analysis of the photometric and spectroscopic data, and we will also compare with the data of Gonzalez-Bedolla to check for possible period or amplitude changes. 


\section{References}

Dall T. H., Frandsen S., Lehmann H., Anupama G. C., Kambe E., Handler G., Kawanomoto S., Watanabe E., Fukata M., Nagae T., Horner S. 2002, A\&A 385, 921 Dall T. H., Frandsen S. 2002, A\&A 386, 964

Gonzalez-Bedolla S. 1990, Rev. Mex. A\&A 21, 401

Gonzalez-Bedolla S., Rolland A., Rodriguez E., Lopez de Coca P. 1990, IBVS 3533

Jørgensen H. E., Johansen K. T., Olsen E. H. 1971, A\&A 12, 223

Sperl M. 1998, Comm. in Asteroseismology 111, 1

Viskum M., Kjeldsen H., Bedding T. R., Dall T. H., Baldry I. K., Bruntt H., Frandsen S. 1998, A\&A 335, 549 